\section{Type 3 confined placental mosaicisms excluding trisomies 16 are also associated with adverse pregnancy outcomes}

\section{To the Editor:}

We read with great interest the article published by Grati et al. entitled "Outcomes in pregnancies with a confined placental mosaicism and implications for prenatal screening using cell-free DNA." " This article investigated the association between confined placental mosaicism (CPM) and adverse pregnancy outcomes. From a large series of 76,102 chorionic villus samplings (CVS), $124 \mathrm{CPMs}$ (including rare autosomal trisomies, tetraploidies, and whole autosomal arm imbalances) of which the pregnancy outcomes were documented were compared with 468 control pregnancies. From this study the authors concluded that only trisomy 16 restricted to the placenta presented an increased risk of adverse pregnancy outcome (birthweight percentile <3rd and spontaneous preterm delivery), while excluding trisomy 16, the pregnancy outcome of CPM for rare autosomal trisomies is close to that observed for control pregnancies. It is therefore an extensive cohort for CPM pregnancy outcomes, and we would like to congratulate the authors on their work.

As mentioned by the authors, the influence of CPM on pregnancy outcome is still a controversial topic. Some previous studies reported that the outcome of pregnancies complicated by CPM appeared to be predominantly favorable, whereas others pointed out that these pregnancies were significantly associated with an increased risk of intrauterine growth restriction and/or intrauterine fetal death. ${ }^{2-10}$

Between 1997 and 2015, we performed more than 19,000 CVS with well-documented cytogenetic examinations and clinical outcomes. We identified that the main parameter able to predict clinical outcome in pregnancies complicated by CPM was the CPM subtype. ${ }^{9,10}$ Precisely, type 1 and type 2 CPM (mainly from mitotic origin) had no effect on fetal development, whereas type 3 CPM (mainly from meiotic origin) was associated with preterm birth, (severely) small for gestational age newborns, and adverse pregnancy outcomes. ${ }^{9,10}$

In the two studies we performed in this field, we took into account common trisomies (trisomies 13, 18, and 21) and rare autosomal trisomies restricted to the placenta, and excluded tetraploidies. ${ }^{9,10}$ In the statistical analysis we performed, we did not separate CPM involving trisomy 16 from common trisomies and other rare autosomal trisomies. ${ }^{9,10}$ In the light of the results reported by Grati et al., ${ }^{1}$ we wished herein to re-examine our data.

When excluding the 14 trisomies 16 confined to placenta from our results, we observed 26 type $3 \mathrm{CPMs}$ with welldocumented cytogenetic examinations and clinical outcomes: one trisomy 2 , one trisomy 4 , one trisomy 5 , one trisomy 6 , three trisomies 7 , one trisomy 8 , two trisomies 9 , one trisomy 13, two trisomies 15 , one trisomy 17 , one trisomy 18 , one trisomy 21 , six trisomies 22 , one trisomy $(2+9)$, and three monosomies X. ${ }^{9,10}$ In these pregnancies, 11 adverse clinical outcomes were described: one termination of the pregnancy for maternal uniparental disomy of chromosome 15 (Prader-Willi syndrome), three intrauterine fetal deaths consecutive to severe fetal growth restriction, six severe neonatal hypotrophies (birthweight percentile $<3^{\text {rd }}$ ), and one perinatal death at 25 days consecutive to severe fetal growth restriction. ${ }^{9,10}$ Consequently, we observed 11/26 (42\%) of adverse clinical outcomes in patients with type $3 \mathrm{CPM}$ excluding trisomies 16 . It also has be noticed that among the 15 patients with a "normal" clinical outcome, 9 neonatal hypotrophies (birthweight percentile $<10$ th) were observed. Collectively, type $3 \mathrm{CPMs}$ excluding trisomies 16 were associated with at least a neonatal hypotrophy in $(11+9) / 26(77 \%)$ of these pregnancies. Regarding type 1 and type 2 CPM, we did not notice any effect on fetal development, which was in agreement with the results reported by Grati et al. ${ }^{1,10}$

Type 3 CPM is the least common subtype of CPM (only $\sim 10 \%$ of CPMs are type 3 ), and unlike Grati et al. ${ }^{1}$ we took into account in our analysis common trisomies restricted to the placenta. In our opinion, these two previous points could explain the contradictory results between those reported by Grati et al. ${ }^{1}$ and by our team. As described herein, type 3 CPMs excluding trisomies 16 are also associated in our experience with adverse pregnancy outcomes and fetal growth restriction, and although rare, these placental chromosomal abnormalities definitely require appropriate obstetrical monitoring. ${ }^{9,10}$

\section{DISCLOSURE}

The authors declare no conflicts of interest.

Publisher's note Springer Nature remains neutral with regard to jurisdictional claims in published maps and institutional affiliations.

\section{Jérôme Toutain, PharmD, $P h D \mathbb{D i D}^{1}$,} Jacques Horovitz, $M D$ (1) $^{1}$ and Robert Saura, $M D \mathbb{1}^{1}$

${ }^{1}$ Centre de Médecine Fotale, Maternité Pellegrin, CHU de Bordeaux, Bordeaux, France Correspondence: Jérôme Toutain (jerome.toutain@chu-bordeaux.fr) 


\section{REFERENCES}

1. Grati FR, Ferreira J, Benn P et al. Outcomes in pregnancies with a confined placental mosaicism and implications for prenatal screening using cell-free DNA. Genet Med. 2019 Aug 8; https://doi.org/10.1038/ s41436-019-0630-y [Epub ahead of print].

2. Goldberg JD, Wohlferd MM. Incidence and outcome of chromosomal mosaicism found at the time of chorionic villus sampling. Am J Obstet Gynecol. 1997;176:1349-1352.

3. Amor DJ, Neo WT, Waters E, et al. Health and developmental outcome of children following prenatal diagnosis of confined placental mosaicism. Prenat Diagn. 2006;26:443-448.

4. Baffero GM, Somigliana E, Crovetto F, et al. Confined placental mosaicism at chorionic villus sampling: risk factors and pregnancy outcome. Prenat Diagn. 2012;32:1102-1108.

5. Kalousek DK, Howard-Peebles PN, Olson SB, et al. Confirmation of CVS mosaicism in term placentae and high frequency of intrauterine growth retardation association with confined placental mosaicism. Prenat Diagn. 1991;11:743-750.

6. Robinson WP, Barrett IJ, Bernard L, et al. Meiotic origin of trisomy in confined placental mosaicism is correlated with presence of fetal uniparental disomy, high levels of trisomy in trophoblast, and increased risk of fetal intrauterine growth restriction. Am J Hum Genet. 1997;60:917-927.

7. Stipoljev F, Latin V, Kos $M$, et al. Correlation of confined placental mosaicism with fetal intrauterine growth retardation. A case control study of placentas at delivery. Fetal Diagn Ther. 2001;16:4-9.

8. Wilkins-Haug L, Quade B, Morton CC. Confined placental mosaicism as a risk factor among newborns with fetal growth restriction. Prenat Diagn. 2006;26:428-432.

9. Toutain J, Labeau-Gaüzere C, Barnetche $T$, et al. Confined placental mosaicism and pregnancy outcome: a distinction needs to be made between types 2 and 3. Prenat Diagn. 2010;30:1155-1164.

10. Toutain J, Goutte-Gattat D, Horovitz J, Saura R. Confined placental mosaicism revisited: Impact on pregnancy characteristics and outcome. PLOS ONE. 2018;13:e0195905.

Advance online publication 1 October 2019. doi:10.1038/s41436-019-0664-1 